\title{
Editorial
}

\section{Lifestyle changes after a myocardial infarction}

A survey in general practice ${ }^{1}$ has shown that $90 \%$ of patients with previously identified coronary heart disease have at least one and usually two or more lifestyle related cardiovascular risk factors such as smoking, poor diet or a sedentary lifestyle. These risk factors are likely to have contributed to their heart disease and, following a myocardial infarction (MI), may inhibit recovery and increase the chance of reinfarction. It is easy to be unduly pessimistic about lifestyle change. Many people change their lifestyle for health reasons. There are more ex-smokers than smokers in the UK and most of these ex-smokers stopped without professional help. On the other hand a sizeable proportion of the population are aware of the dangers of a high fat diet without apparently being either willing or able to do much about it.

It has been know for some time that many smokers stop smoking after an MI; perhaps as many as $45 \%$ of patients, which is between two and four times the success rate of antismoking clinics for the public in general. However the position is less satisfactory for other risk factors. Patients might become less active after an MI (partly because of the consequent physical impairments and change in work patterns). While there is some evidence that patients reduce self reported saturated fat intake in the year following an MI these changes can be small and are often not reflected in changes in lipid levels. Despite this, immediately after an $\mathrm{MI}$ is a good time to attempt to change behaviour. People change their behaviour because of some significant change in their life or their thinking. An MI is for most patients a major life threatening event that they, and those dealing with them, frequently ascribe to features of their lifestyle. They are therefore very likely to wish to alter their behaviour. The medical and related personnel treating them have considerable authority shortly after an MI and their advice is likely to be given unusual weight. This authority should be used by giving clear relevant advice. If such advice is not given, not only is an opportunity missed but also the lack of advice may be interpreted as sanctioning the high risk behaviour.

\section{Standard behavioural risk factors}

While many patients will change their lifestyle with little or no professional assistance, other than information and advice, some will require systematic help. Many programmes have been developed and tested on the general population to reduce smoking or weight, or to alter diet. However it cannot be assumed that what works in the healthy individual who decides that they wish to change their behaviour will work with the post-MI patient who is told to change but who may not be convinced of the need. Nevertheless, it is reasonable to assume that intensive behavioural programmes developed for the general population, which may involve group meetings on a weekly basis for two or three months, will produce behaviour change in patients who intend to change their behaviour. Aspects of such programmes could be, and occasionally are, incorporated into cardiac rehabilitation programmes. However, the existing provision of cardiac rehabilitation is weak and the uptake very selective. A better model may be nurse managed programmes that start while the patient is still in hospital. DeBusk and colleagues have been developing such a model for many years ${ }^{23}$; this model has been particularly effective for smoking cessation. An inpatient programme that supplements a vigorous antismoking message (and a non-smoking hospital policy) with manual based training in avoiding relapse and telephone contact in the few months following discharge, increased 12 month smoking cessation to over $70 \%$. Attempts to use a similar case management approach to aid dietary change have been less successful and it is likely that pharmacological methods will be the main method of lipid control in patients with very high lipid levels (although a very intensive and strict dietary and exercise regimen did lead to reductions in blood cholesterol and a reduction in coronary artery stenosis in patients with coronary artery disease ${ }^{4}$. Home based exercise programmes based on the patients' preferred activity (walking, swimming, cycling, etc) are acceptable to most patients and lead to improved functional capacity. ${ }^{2}$ Such programmes obviously have to be implemented with care and involve careful assessment of the patient's capacities and self monitoring of heart rate during exercise. The best of modern rehabilitation programmes also tackle many aspects of risk factor change. They are also likely to be effective if comprehensive and if, as Thompson and de Bono recommend in another editorial in this series, ${ }^{5}$ the interventions are designed to transfer seamlessly from hospital to community care. Some of the difficulties in effecting this transfer were demonstrated in the recent SHIP trial (Southampton heart integrated care project) of the provision of specialist cardiac liaison nurses. ${ }^{6}$

\section{Stress}

While the potential value of altering lifestyles associated with the main risk factors for cardiovascular disease is widely accepted, the value of altering other less well established risk factors is more contentious. Most patients claim that their heart attack was stress related; however, while the evidence linking stress or stress related behaviours such as anger and hostility and cardiovascular disease is strengthening, it is not conclusive. Nevertheless, such stress related processes may play a role in both arterial disease and, even more importantly, in triggering acute MI through the effects of acute stress and the associated emotions on plaque rupture and thrombogenesis. ${ }^{7}$ While there are negative reports it seems clear that brief psychosocial interventions delivered as part of rehabilitation both before discharge from hospital or on an outpatient basis can reduce the anxiety and depression associated with an $\mathrm{MI}^{8}$ and perhaps reduce reinfarction rates. ${ }^{9}$ Enduring characteristics such as hostility can also be altered, but this involves specialist cognitive behavioural interventions that are unlikely to be used routinely but should be considered for the exceptionally stressed, hostile or explosively angry patient ${ }^{10}$ who may well mention such behaviours if questioned about their lifestyle by a cardiologist or specialist cardiac nurse. While interventions to reduce stress or hostility have been shown to reduce reinfarction rates, ${ }^{10}$ further studies are required to establish their general applicability and value. At present it is probably most reasonable to regard reducing stress and the associated 
distress as improving the patient's quality of life rather than necessarily reducing the risk of reinfarction.

\section{Adherence to medical regimens}

For many patients the main and probably the most critical lifestyle change following an $\mathrm{MI}$ is a chronic medication regimen; adherence to such medication is poor. Only $10 \%$ of elderly patients with congestive heart failure obtain the prescribed dosage of medication, ${ }^{11}$ while even in middle aged populations on simple treatment regimens, adherence to aspirin or lipid lowering drugs can be less than $50 \%$. In a recent systematic review $^{12}$ it was shown that nonadherence to medication has a substantial effect on disease outcome in coronary heart disease, although the effect may not be entirely pharmacological as non-adherence to placebo is also associated with a poor outcome. Perhaps surprisingly there appears to have been little systematic work on improving adherence with medication post-MI; however, in other conditions, such as cancer, nurse led educational programmes improve adherence and medical outcomes. Such programmes usually involve explaining the purpose of the medication, self monitoring, simplifying the regimen or its administration, and giving training in taking the medication appropriately. ${ }^{13}$ The latter may be particularly important for elderly patients who are often on very complex treatment regimens.

\section{What to alter, and in whom?}

Altering lifestyle has at least two components, the patient has to decide to alter some relevant aspects of their lifestyle, and they must have the capacity to do this. Different procedures are required for the different components. This is seen most clearly with smoking cessation where success is rare with patients who do not intend to give up smoking but very high with patients who do. The empirical literature does not offer much evaluated guidance on how to change intentions. Therefore it is most effective to concentrate on improving the capacity of patients who intend to alter their behaviour. There is compelling evidence that such patients benefit from assistance in giving up smoking, and empirical support for programmes to increase exercise and reduce stress and anxiety. This assistance involves giving them the necessary skills and offering the back-up needed to maintain motivation. Nurse led inpatient programmes followed by telephone contact is a feasible and successful model. The comprehensive rehabilitation programmes described by Thompson and De Bono ${ }^{5}$ are also attractive approaches.

School of Psychology, University of St Andrews

D W JOHNSTON

St Andrews, Fife KY16 9fU, UK

1 Campbell NC, Thain J, Deans HG, et al. Secondary prevention in coronary heart disease: baseline survey of provision in general practice. $B M \mathcal{F}$ 1998;316:1430-4.

2 DeBusk RF, Miller NH, Superko R, et al. A case-management system for coronary risk factor modification after acute myocardial infarction. Ann Intern Med 1994;120:721-9.

3 Taylor CB, Miller NH, Killen JD, et al. Smoking cessation after acute myocardial infarction: effects of a nurse managed intervention. Ann Intern Med cardial infarction:

4 Ornish D, Brown SE, Scherwitz LW, et al. Can lifestyle change reverse coronary heart disease? Lancet 1990;336:129-33.

5 Thompson DR, de Bono DP. How valuable is cardiac rehabilitation and who should get it? Heart 1999;82:545-6.

6 Jolly K, Bradley F, Sharp S, et al. Randomised controlled trial of follow-up care in general practice of patients with myocardial infarction and angina: final results of the Southampton heart integrated care project. BMF 1999; 318:706-11.

7 Muller JE, Abela GS, Nesto RW, et al. Triggers, acute risk factors and vulnerable plaques: the lexicon of a new frontier. 7 Am Coll Cardiol 1994;23:809-13.

8 Johnston M, Foulkes J, Johnston DW, et al. The impact on patients and partners of inpatient and extended counselling and rehabilitation: a controlled trial. Psychosom Med. 1999;61:225-33.

9 Linden W, Stossel C, Maurice J. Psychosocial interventions for patients with coronary artery disease. Arch Intern Med 1996;156:745-52.

10 Burell G, Ohman A, Sundin O, et al. Modification of the type A behaviour pattern in post-myocardial infarction patients: a route to cardiac rehabilitation. Int $\mathcal{f}$ Behavioral Med 1994;1:32-54.

11 Mohane M, Bohn RL, Gurwitz JH, et al. Noncompliance with congestive heart failure therapy in the elderly. Arch Intern Med 1994;15:433-7.

12 McDermott MM, Schmitt B, Wallner E. Impact of medication nonadherence on coronary heart disease outcomes: a critical review. Arch Intern Med 1997;157:1921-9.

13 Miller NH, Hill M, Kottke T, et al. The multilevel compliance challenge: recommendations for a call to action. Circulation 1997;95:1085-90. 